\title{
CXCR4 HAS THE POTENTIAL TO ENHANCE BONE FORMATION IN OSTEOPENIC RATS
}

Sanghani, Anita PhD¹, Osagie-Clouard, Liza MD¹, Samizadeh Soureouseh PhD¹, Coathup Melanie PhD'1, , Kalia, Priya PhD1, Di Silvio, Lucy PhD², Blunn, Gordon PhD'

Works performed at:

${ }^{1}$ Institute of Orthopaedics and Musculoskeletal Science, University College London

${ }^{2}$ Department of Biomaterials and Biomimetics, Kings College London

Corresponding Author

Liza Osagie-Clouard

IOMS, RNOH, Brockley Hill, Middlesex HA7 4LP

$+447931809218$

l.osagie@ucl.ac.uk

Author contacts:

a.sanghani.12@ucl.ac.uk

s.samizadeh@ucl.ac.uk

m.coathup@ucl.ac.uk

p.kalia@ucl.ac.uk

g.blunn@ucl.ac.uk

All contactable at

IOMS, RNOH, Brockley Hill, Middlesex HA7 4LP

+44 2089095887, fax +442089095886 
Page 2 of 26

I.disilvio@kcl.ac.uk

Floor 17,

Tower Wing

Guy's

London, United Kingdom

+442078488475 


\section{Abstract}

Osteoporosis is characterised by reduced bone mass and aberrant bone microarchitecture; thus increasing susceptibility to fracture due to reduced strength and quality. The aims of this study were to investigate the role of CXCR4 transfected on stem cell homing and osteogenic characteristics in osteopenic rats; particularly elucidating the effect on cell migration.

Methods: MSCs were harvested from young, adult and ovarectomized animals and transfected with CXCR4; these cells were administered intravenously in ovarectomized rats. Micro CT and mechanical testing was completed after 12 weeks.

Results: rats injected with young CXCR4 transfected cells had significantly higher BMD compared to placebo injected rats $(p<0.05)$. Rats injected with ovarectomized CXCR4 transfected cells, had higher BMD compared to those injected with saline or nontransfected cells $(p<0.04)$. L4 vertebral stiffness was significantly higher in rats treated with young CXCR4 transfected cells compared to all other groups $(p<0.05)$.

Conclusions: CXCR4 genetically modified cells from young and ovarectomized sources improve some aspects of bone formation in the ovarectomized model of osteoporosis; and thus, may play a role in patient treatment regimens. 


\subsection{Introduction}

Post-menopausal oestrogen deficiency leads to an uncoupling of the bone remodelling cycle, whereby upregulated osteoclast activity, goes either unmatched or is coupled with aberrant osteoblast activity; thus leading overall net bone resorption. Defined as a skeletal disorder characterised by reduced bone strength and thus increased fracture risk (1); type one oestrogen deficient osteoporosis is a result of both a reduction in bone density and bone quality.

Long term treatments aim to improve both bone strength and quality, be they anabolic or anti-catabolic in nature, interventions aim to in part restore a balance remodelling cycle (2); yet, as well as problems inherent to osteoporosis alone, the patient age will also hinder remodelling and regeneration efficacies. Bone formation may be compromised due to the number of mesenchymal stem cells (MSCs) within bone marrow diminishing with age $(\underline{3-6})$, the ability of these cells to differentiate into osteoprogenitor cells and mature osteoblasts ( $\underline{7-10})$, and the ability of MSCs from aged sources to mobilise from their niche, home across the tissue endothelium and mature into active cell types that modulate the fracture environment. Bone healing following injury is in part modulated by quiescent MSCs at the fracture site, but also by the ability of latent cells to home/migrate to the site of injury; as such a number of preclinical studies have investigated the role of transplanted MSCs in fracture healing and in increasing bone mass (11). For a MSC transplantation to generate a clinical benefit, MSCs must engraft at the site of bone injury or the bone marrow. Chemokines play significant roles in controlling cellular migration; Granero-Molto identified the importance of the stromal derived factor-1/chemokine receptor type 4 (SDF$1 /$ CXCR4) axis in modulating cell migration from bone marrow. SDF-1/(1ㅗ $\underline{12})$. Transplantation of MSCs over-expressing RANK Fc(an antagonist of RANK L) or CXCR4 (13) as well as, CXCR4 and Cbfa1 (14) have been shown to restore bone mass and strength in osteopenic mice. In both these studies, MSCs were intravenously introduced into the mice through the tail vein, and MSCs over-expressing CXCR4 promoted in vivo cell trafficking to bone in OVX mice. This therapeutic effect of CXCR4 transfected MSCs therefore protects against bone loss and increases bone formation. 
In this study we make a comparison of bone formation when MSCs from young rats and OVX rats transfected with CXCR4 as well as un-transfected OVX MSCs was used in an osteopenic rat model. The comparison between the two cell types investigates the beneficial effects of autologous cells from osteoporotic patients and may allude to the efficacy of CXCR4 modification in osteoporotic treatments; as such, we hypothesis that CXCR4 transfected MSCs when administered intravenously, would migrate to the bone marrow and improve bone formation in an osteopenic rat model of osteoporosis.

\subsection{Methodology}

\subsubsection{Animal model}

6 to 9-month old female ex-breeder wistar rats were purchased following ovariectomy. At 16 weeks post-ovariectomy and following euthanasia, femoral bone mineral density was measured using peripheral quantitative computed tomography pQCT (Stratec XCT1000) to confirm osteopenia and this was compared with control non-ovariectomised rats. As such, cells from these animals were used experimentally at 10-13months

\subsubsection{Cell culture}

Rat bone marrow mesenchymal stem cells (rBMCs) were harvested from 2-4 week old young and the aforementioned ovariectomised Wistar rat femora ( $n=6$ per group). Bone marrow cells were harvested by flushing the femora with Dulbecco's modified Eagle medium (DMEM), 20\% fetal calf serum, 1\% Penicillin Streptomycin (P/S) in a $25 \mathrm{~cm}^{2}$ flask. Cells were cultured at $37^{\circ} \mathrm{C}$ at $5 \% \mathrm{CO}_{2}$. Cells were characterised using tri-lineage differentiation and flow cytometry and used at passage 3.

\subsubsection{Preparation of the recombinant adenoviruses}

The human CXCR4 CDNA (pCMV-XL5-CXCR4 Origene) was digested by restriction endonucleases Notl and Xhol and then inserted into pShuttle-CMV (AdEasy XL Adenoviral vector system; Stratagene) to form pShuttle-CMV-CXCR4. The human CXCR4 gene and the pShuttle-CMV vector were both cut by restriction endonuclease Notl and Xhol and were then repaired to form blunt ends. Human CXCR4 CDNA was then cloned into the pShuttleCMV vector using DNA ligase. The presence of the insert was confirmed by restriction digest as well as Sanger sequencing. The incorporated shuttle vector was then linearised 
with Pmel restriction endonuclease and transformed into BJ5183-AD-1 competent cells using electroporation. A scrambled plasmid was created by inserting no gene into the pShuttle-CMV vector.

Following selection of the smallest colonies, the recombinant plasmid was collected and cut using the Pacl restriction enzyme. The correctly identified copies were then largely expanded in bulk using the recombinant-deficient XL10-Gold strain. Purified recombinant plasmid DNA, cut with Pacl was then used to transfect AD-293 cells. The viral particles were generated by alternate freezing and thawing of the infected AD293 cells and collecting the supernatant. An anti-hexon antibody stain was used to determine the plaque forming units $/ \mathrm{ml}(\mathrm{pfu} / \mathrm{ml})$ of the infected cells by counting the number of dark infected cells.

\subsubsection{Analysis of CXCR4 over-expression on infected cells}

Young and OVX MSCs from the third passage were infected with the CXCR4 adenovirus at a MOI of 800, trypsinised and centrifuged at $2000 \mathrm{rpm}$ for 10 minutes before being resuspended at 100,000 cells in phosphate buffered saline (PBS). Cell aliquots were permeabilised in ice cold methanol for 10 minutes and incubated with primary CXCR4 antibody (Abcam) for 1 hour at room temperature. The cells were washed in PBS and then incubated in secondary goat anti-rabbit antibody (Abcam) for 30 minutes at room temperature. The negative control consisted of cells incubated in the secondary antibody only. 100,000 cells were then analysed using a flow cytometry machine (Cytoflex, Beckman Coulter). The results were compared to uninfected MSCs from the same rats.

\subsubsection{Intravenous administration of CXCR4 transfected MSCS}

30 female ovariectomised rats (average weight $494.2 \pm 53.8 \mathrm{~g}$ ) were injected with $0.5 \mathrm{ml}$ Sodium Nitroprusside $(25 \mathrm{mg} / \mathrm{ml})$, followed by 1 million cells suspended in $2 \mathrm{ml}$ sterile saline and finally $0.2 \mathrm{ml}$ of saline to clear any cells left in the syringe. Successful injections were confirmed when the fluid was injected without any resistance and there was no swelling of the tail. The rats in this study were divided into two parts (Figure 6.1): 
a. Cell tracker group: 6 rats were injected with Vybrant-1,1'-Dioctadecyl-3,3,3',3'Tetramethylindocarbocyanine Perchlorate (Dii) labelled cells (ThermoFisher Scientific, UK). This group of rats was used to track the cells 7 days after injection in order to illustrate effective mobilisation.. This was the 'cell tracker group' (Day 0 control rats). The rats were euthanised 7 days post-injection and the samples retrieved from these rats were also used to obtain baseline histological, micro/pQCT and mechanical data. The rats were divided into 2 groups:

- Dil labelled Young MSCs + Empty Plasmid $(n=3)$

- Dil labelled Young MSCs + CXCR4 $(n=3)$

To investigate the homing ability of MSCs, cells were labelled with Dil cell-labelling solution $1 \times 10^{6}$ and plated in a T225 flask. 100ul of the Dil solution was suspended in $5 \mathrm{ml}$ of serum free media, cells were incubated in this for 30minutes at 37C.

The rats were euthanised 7 days (Day 0 controls) post-injection and their femurs and tibias retrieved. The bone specimens were fixed in $10 \%$ buffered Formaldehyde, and decalcified in $12.5 \%$ ethylenediaminetetraacetic acid (EDTA), $\mathrm{pH}$ 8. Decalcification was confirmed by radiography, after which the specimens were dehydrated in increasing alcohol concentrations, treated with chloroform for 2 days to de-fat the tissue and then embedded in wax. Sections measuring $5 \mu \mathrm{m}$ thick were made using a microtome (Shandon Finesse 325, ThermoFisher Scientific, UK).

Sections were de-waxed twice in xylene, placed in two changes of $100 \%$ alcohol and then hydrated in serial dilutions of alcohol. After hydration, samples were stained in haematoxylin, a nuclear stain, for 5-10 minutes. The excess stain was washed off by immersing the slides in water for 5 minutes. Samples were then differentiated in $0.5 \% \mathrm{HCL}$ acid (made up in $70 \%$ alcohol) and washed using water. After removing the acid-alcohol, samples were counterstained in $1 \%$ eosin for 3-4 minutes, washed in water and dehydrated by serial dilutions of alcohol. Finally, samples were cleaned by xylene and mounted under coverslips using Pertex Mounting Medium (CellPath plc, UK). Samples were observed under a light microscope (KS-300 Zeiss, UK). This was used to observe the Dil labelled cells that had migrated to the bone (See section 6.2 .6 below). The H\&E images 
observed under a light microscope allowed the location of the fluorescent cells to be identified in the bone. Slices were then imaged under a fluorescent microscope (Zeiss Apatome 2). The slides were stained with Hoechst 3342 (Thermofisher Scientific, UK) and mounted with Fluoromount ${ }^{\mathrm{TM}}$ Aqueous Mounting Medium (Sigma Aldrich, UK) and viewed under an emission wavelength of $617 \mathrm{~nm}$.

b. Experimental treatment group: OVX rats were divided into 4 groups; Groups A, B and $C$ were injected with cells and Group $D$ received saline injection. The rats in this group were euthanized after 11 weeks. The treatment groups were divided as follows:

- Group A - Young MSCs + CXCR4 (n=6)

- Group B - OVX MSCs (n=6)

- Group C-OVX MSCs + CXCR4 (n=6)

- Group D - Saline $(n=6)$

From all the rats in both groups, the following samples were retrieved; the femora, tibiae, left humerus and L4 and L5 vertebrae for mechanical testing, micro CT and pQCT only..

\subsubsection{Vertebral compression}

L4 and L5 vertebra were separated from the rest of the vertebral column by halving the discs and cutting the ligaments. The facet joints and spinous processes were preserved to maintain physiological motion of the vertebrae during compression. Each vertebra was individually compressed at a speed of $0.75 \mathrm{~mm} / \mathrm{min}$ using a Zwick LFM machine, until failure and the ultimate compressive strength was measured.

\subsubsection{Three-point bending test of humerus, tibia and femur}

From the retrieved long bones all tissue and muscle attachments were cleared. The length of the bones was measured, the loading pin was set up at the bone's mid shaft and the bones were loaded at a speed of $0.5 \mathrm{~mm} / \mathrm{min}$ using a Zwick LFM machine, until failure point when the bone fractured. The span between the two lower supports was set $10 \mathrm{~mm}$ apart. A force displacement graph was generated and used to calculate the stiffness and maximum load of the bones manually. 


\subsubsection{Peripheral Quantitative Computed Tomography (pQCT) of the tibia and femur}

The skin and soft tissue around the right and left femur were removed and BMD measured within the femoral condyles using PQCT (XCT 2000, Stratec, Pforzheim, Germany). The samples were fixed in formalin before scanning and were placed in a sealed $50 \mathrm{ml}$ Falcon tube during the scan. The total length of the bone was obtained prior to scanning and the femoral head of each sample was scanned symmetrically at slice intervals of $0.5 \mathrm{~mm}$. 3 slices were acquired at $10 \%$ and 1 slice was acquired at $20 \%$ (of the femoral length) from the proximal femur end. The samples were analysed using XCT software.

\subsubsection{MicroCT of the femoral metaphysis}

Samples were transported in PBS and wrapped in cling film for scanning (SkyScan 1172, Bruker, Belgium). The $x$-ray source was set at $70 \mathrm{kV}$ with a current of $120 \mathrm{uA}$. To reduce beam hardening, a $0.5 \mathrm{~mm}$ aluminium filter was used during the scan. During the scan a rotation degree of $0.6^{\circ}$, a frame averaging of 2 and a voxel size of 6um was applied. The NRecon software (Bruker, Belgium) was used to reconstruct the scanned images with the following parameters: smoothing $=2$, beam hardening factor correction $=12 \%$ and ring artefact reduction $=41 \%$.

The trabecular bone was analysed using a CtAn software (Bruker, Belgium). The growth plate was selected as a reference point and a volume of 150 slices were selected, 200 slices above the growth plate (Figure 2). For analysis, a volume of interest (VOI) was chosen by selecting the metaphyseal trabecular region and excluding the cortical bone and this region was used to measure the Bone Volume (BV) and Bone surface (BS).

\subsubsection{Statistical analysis}

The normality was checked using a Shapiro Wilkinson test and found to be nonparametric; as such, comparisons were made using a Mann-Whitney $U$ test with a Bonferroni correction. All data was analysed using SPSS version 24 (Chicago, USA). 


\subsection{Results}

\subsubsection{Establishment of the osteopenic rat model}

The mean femoral BMD measured in the OVX rat group was significantly lower $(538.2 \pm$ $\left.23.3 \mathrm{~g} / \mathrm{cm}^{3}\right)$ when compared with control rats $\left(666.9 \pm 46 \mathrm{~g} / \mathrm{cm}^{3}\right)(p=0.0002)$. BMD was seen to reduce by $19.3 \pm 9 \%$.

\subsubsection{CXCR4 expression of infected and uninfected cells}

After transfection with the CXCR4, $92 \pm 2.6 \%$ and $40 \pm 16.1 \%$ of young and OVX MSCS respectively over-expressed CXCR4. CXCR4 expression significantly increased $(p=<0.05)$ from $19.4 \pm 9.8 \%$ in non-transfected OVX cells to $40.0 \pm 16.1 \%$ in transfected cells. In young MSCs CXCR4 expression only increased by $5 \%$ after transfection although non transfected young MSCs expressed high endogenous levels of CXCR4 (Figure 3).

\subsubsection{Fate of systemically injected MSCs}

At day 7, numerous Dil labelled nucleated cells were observed in the blood vessels in the cortical and trabecular bone of the femoral condyles, confirmed on $\mathrm{H}+\mathrm{E}$ and fluorescent imaging (Figure 4). No fluorescent cells were visible in slides from rats that were injected with unlabelled cells.

\subsubsection{Vertebral compression}

The stiffness of the L4 vertebrae was significantly higher for the group that received the Young-CXCR4 MSCs $(251.5 \pm 61.7 \mathrm{~N} / \mathrm{mm})$ in comparison to the rats that received a sham saline injection (99.2 $\pm 16.2 \mathrm{~N} / \mathrm{mm}$ ) (Figure 5).

\subsubsection{Three-point bending test of the humerus, tibia and femur}

The stiffness of the humerus, tibia and femur were obtained by calculating the gradient of the force displacement graph. No significance difference was seen between the different groups. However, the rats receiving the saline injections had the lowest stiffness compared to those that received CXCR4-OVX MSCs and CXCR4-young MSCs. 


\subsubsection{PQCT of the tibia and femur}

Young MSCs transfected with CXCR4 significantly improved the BMD of the bone in the femoral condyles of the OVX rats. This significant difference in BMD was seen for the trabecular bone, cortical/subcortical bone and total BMD (Figure 6).

There was no significant difference between OVX rats injected with young MSCS transfected with CXCR4 or injected with saline (transfected-trabecular BMD of $716.4 \pm$ $105.7 \mathrm{mg} / \mathrm{ccm}$, total BMD of $694 \pm 80.1 \mathrm{mg} / \mathrm{ccm}$ and Cort/sub cort BMD of $715.2 \pm 45.4$ $\mathrm{mg} / \mathrm{ccm}$, saline-trabecular BMD of $535.5 \pm 61.6 \mathrm{mg} / \mathrm{ccm}, 563.4 \pm 82.9 \mathrm{mg} / \mathrm{ccm}$ and $576.2 \pm 11$ $\mathrm{mg} / \mathrm{ccm}$ respectively.

\subsubsection{Micro CT and Histomorphometric Analysis of the metaphysis}

The effect of stem cell injection on the bone volume was subtle compared to saline injection but the groups injected with saline and the OVX group (the group prior to any intervention) showed fewer inter-connected trabeculae compared to the animals injected with transfected young stem cells (Figure 7).

Rats treated with young-CXCR4 MSCs had the highest BS area $\left(9.5 \times 10^{7} \pm 5.3 \times 10^{7} \mu \mathrm{m}^{2}\right)$ compared to rats treated with OVX-CXCR4 MSCs $\left(7.6 \times 10^{7} \pm 5.0 \times 10^{7} \mu \mathrm{m}^{2}\right)$, OVX MSCs $\left(7.1 \times 10^{7} \pm 1.7 \times 10^{7}{\mu \mathrm{m}^{2}}^{2}\right)$ and saline $\left(7.3 \times 10^{7} \pm 5.5 \times 10^{7} \mu \mathrm{m}^{2}\right)$. As expected the Time 0 controls had the highest bone surface area because they were euthanised 7-days postinjection while the other groups of rats were euthanised 5-weeks post-injection.

Of the experimental groups, rats injected with saline $(p=0.03)$, OVX MSCs $(p=0.004)$ and OVX-CXCR4 ( $p=0.02$ ) MSCs had a significantly lower bone surface area compared to the rats injected with Young-CXCR4 MSCs ( $p=0.07$ ) (Figure 8).

In all groups injected with stem cells bone volume (BV) was increased in comparison to saline injection. The rats treated with OVX-CXCR4 MSCs had the highest BV $\left(1.7 \times 10^{9} \pm 1.4\right.$ $\left.\times 10^{9} \mathrm{~mm}^{2}\right)$ compared to rats treated with Young-CXCR4 MSCs $\left(1.5 \times 10^{9} \pm 7.0 \times 10^{8} \mu \mathrm{m}^{2}\right)$, $\operatorname{OVX} \operatorname{MSCs}\left(1.4 \times 10^{9} \pm 2.8 \times 10^{8} \mu \mathrm{m}^{2}\right)$ and saline $\left(1.1 \times 10^{9} \pm 8.5 \times 10^{8} \mu \mathrm{m}^{2}\right)$. 


\subsection{Discussion}

The hypothesis of this study was that CXCR4 transfected MSCs when administered intravenously, would migrate to the bone marrow and improve bone density and architecture. In this paper we reinforced the effective migration of intravenously labelled MSCs to long bones, as well as the poor CXCR4 expression as well as migration of MSCS from OVX rat, we further demonstrated that injecting young MSCs transfected with CXCR4 significantly enhanced BMD and $\mathrm{L} 4$ vertebral strength compared to rats who received placebo saline intravenously (Figures 5 and 6). It was also highlighted that injection of OVX and OVX-CXCR4 MSCs helped improve the BMD and trabeculae structure in the femoral condyles of the OVX rats compared to controls. These findings allude to possible applications of modified stem cells to enhance the bone properties in osteoporotic populations. The deteriorating changes of bone in the OVX rats injected with saline was reflected by the thinning of the trabeculae, reduced bone surface area and bone volume in the proximal femur. These micro-structural changes are common problems in osteoporotic patients, causing fragility fractures $(15,19)$. The small sample number of only 6 rats per group means that trends towards increased trabecular thickness in the femora of OVX rats injected with CXCR4 modified cells, were not clinically significant compared to untreated counterparts. Thus one wonders, if a larger number may actually prove significance, and thus add to the weight of evidence suggesting the utility of CXCR4 modification.

The deteriorating bone structure of the OVX rat model was clearly observed as the Time 0 control rats had the most bone volume and density compared to the rest of the OVX rats which were treated for a further 10 weeks. However, the rats injected with cells had a higher bone surface area and volume compared to the rats injected with saline. There was an increased BMD in the animals injected with cells using PQCT which seemed to contradict the modest increase in bone volume observed by microCT. This is similar to results by Huang and co-workers, 2016 that injected allogeneic MSCs from 8 week old rats into OVX rats and looked at their bone formation. They concluded that although the systemic transfusion of allogeneic MSC is a safe procedure, administering cells only may not be the most effective procedure for preventing osteoporosis. They also observed an increase in BMD using PQCT but no difference in trabeculae structure using microCT (20). 
These differences could be because PQCT measures global bone formation while microCT is more specific as it measures slices specifically for analysing trabeculae structure.

Regenerating the trabeculae to provide structural competence may not be difficult especially where continuity has been lost. Cunningham et al, 2005 investigated the recovery of the trabeculae in the right os-calcis in an ovine model. After removal of the trabeculae in the os-calcis animals were returned to their normal daily activities. However, the trabeculae did not regenerate and the os-calcis remained hollow but with increased density in the cortical bone. This could be because the load was distributed to the upper and lower beams of the bone, hence the increase in density and thickness of the cortical bone (21-23). Once interconnectivity between trabeculae has been compromised it is difficult to reform cross-links because of the mechanisms of load transfer which is altered due to reduced number of bridging elements.

Injecting MSCs, irrespective of the fact that they were from OVX or young rats or uninfected or infected protected against bone loss associated with ovariectomy in rats compared to rats injected with saline. These results are similar to those reported by Lien and co-workers who injected CXCR4-infected cells in OVX rats and observed increased BMD in rats injected with cells compared to sham injections (13). However, they showed even more improved bone formation when they injected cells co-transfected with cbfa- 1 . Sheyn and co-workers, 2016 injected a combinational therapy of MSCs and PTH and showed that PTH aided with the migration of MSCs and also played an anabolic role in bone formation in vertebral defects in pigs (24). This highlights the importance of improving migration of cells and also stimulating their ability to form bone. It is important for cells to home and engraft into the marrow cavity (25) as well as help with the bone loss. Cho and co-workers, 2009 highlighted the role of transfecting the cells with RANK-Fc to improve the BMD in ovariectomised mice and also improved their homing capability to the bone marrow $(14,25)$. Studies by Lien and Cho showed significantly improved BMD after injection of MSCs, however these results were based on PQCT data and unlike this work did not look at changes in the trabeculae using MicroCT. Additionally, they induced osteopenia in younger mice (around 3-4 months old) compared to this study where rats at 
the time of experiments were upto 13 months old; as such delineations are required between the sole effect of increased age as well as osteopenia.

Although significant differences were seen in this study, there were a number of trends noted that were not significant. The number of significant differences seen in this study may have been increased by increasing the number of animals in each group.

However, the novelty and significance of the work in this study is that it investigates the prospect of injecting autologous stem cells in osteoporotic patients. Injecting OVX-MSCs may not be as beneficial as injecting Young-MSCs or Young-CXCR4 MSCs, but we still found trends towards improvements in bone formation parameters with cells derived from ovarectomized rats. As such, our results indicate the possible clinical utility of stem cells from both young and osteopenic populations and may be further enhanced by the modification of CXCR4 expression. Further work is required to fully elucidate this effect, and should also assess in more detail the fate of injected cells-assessing the percentage retained at other sites through haematogenous spread. Ultimately, this work builds on the body of literature supporting stem cell use in bone regeneration and the promise modified cells provide

\subsection{Acknowledgements}

This study was funded by Orthopaedic Research UK (ORUK) and John Scales Endowment Fund. 


\subsection{References}

1. Lelovas PP, Xanthos TT, Thoma SE, Lyritis GP, Dontas IA. The laboratory rat as an animal model for osteoporosis research. Comparative medicine. 2008;58(5):424-30.

2. Ammann P, Rizzoli R. Bone strength and its determinants. Osteoporosis International. 2003;14(3):13-8.

3. Lane NE. Epidemiology, etiology, and diagnosis of osteoporosis. American journal of obstetrics and gynecology. 2006;194(2 Suppl):S3-11.

4. Lippuner K. The future of osteoporosis treatment - a research update. Swiss medical weekly. 2012;142:w13624.

5. Chen XD, Dusevich V, Feng JQ, Manolagas SC, Jilka RL. Extracellular matrix made by bone marrow cells facilitates expansion of marrow-derived mesenchymal progenitor cells and prevents their differentiation into osteoblasts. Journal of bone and mineral research : the official journal of the American Society for Bone and Mineral Research. 2007;22(12):1943-56.

6. Katsara O, Mahaira LG, Iliopoulou EG, Moustaki A, Antsaklis A, Loutradis D, et al. Effects of donor age, gender, and in vitro cellular aging on the phenotypic, functional, and molecular characteristics of mouse bone marrow-derived mesenchymal stem cells. Stem Cells Dev. 2011;20(9):1549-61.

7. Sethe S, Scutt A, Stolzing A. Aging of mesenchymal stem cells. Ageing research reviews. 2006;5(1):91-116.

8. Brack AS, Rando TA. Intrinsic changes and extrinsic influences of myogenic stem cell function during aging. Stem cell reviews. 2007;3(3):226-37.

9. Muschler GF, Nitto H, Boehm CA, Easley KA. Age- and gender-related changes in the cellularity of human bone marrow and the prevalence of osteoblastic progenitors. Journal of orthopaedic research : official publication of the Orthopaedic Research Society. 2001;19(1):117-25.

10. Wang Z, Goh J, Das De S, Ge Z, Ouyang H, Chong JS, et al. Efficacy of bone marrowderived stem cells in strengthening osteoporotic bone in a rabbit model. Tissue Eng. 2006;12(7):1753-61.

11. Sanghani-Kerai A, McCreary D, Lancashire H, Osagie L, Coathup M, Blunn G. Stem 
Cell Interventions for Bone Healing: Fractures and Osteoporosis. Curr Stem Cell Res Ther. 2018 Apr 10 (EPub)

12. Yellowley C. CXCL12/CXCR4 signaling and other recruitment and homing pathways in fracture repair. BoneKEy reports. 2013;2(3).

13. Lien CY, Chih-Yuan Ho K, Lee OK, Blunn GW, Su Y. Restoration of Bone Mass and Strength in Glucocorticoid-Treated Mice by Systemic Transplantation of CXCR4 and Cbfa-1 Co-Expressing Mesenchymal Stem Cells. Journal of Bone and Mineral Research. 2009;24(5):837-48.

14. Cho SW, Sun HJ, Yang J-Y, Jung JY, An JH, Cho HY, et al. Transplantation of mesenchymal stem cells overexpressing RANK-FC or CXCR4 prevents bone loss in ovariectomized mice. Molecular Therapy. 2009;17(11):1979-87.

15. Chen H, Zhou X, Fujita H, Onozuka M, Kubo K-Y. Age-related changes in trabecular and cortical bone microstructure. International journal of endocrinology. 2013;2013.

16. De Souza RL, Matsuura M, Eckstein F, Rawlinson SC, Lanyon LE, Pitsillides AA. Noninvasive axial loading of mouse tibiae increases cortical bone formation and modifies trabecular organization: a new model to study cortical and cancellous compartments in a single loaded element. Bone. 2005;37(6):810-8.

17. Laib A, Kumer J, Majumdar S, Lane N. The temporal changes of trabecular architecture in ovariectomized rats assessed by MicroCT. Osteoporosis International. 2001;12(11):936-41.

18. Bala Y, Bui QM, Wang XF, luliano S, Wang Q, Ghasem-Zadeh A, et al. Trabecular and cortical microstructure and fragility of the distal radius in women. Journal of Bone and Mineral Research. 2015;30(4):621-9.

19. Kiernan J, Hu S, Grynpas MD, Davies JE, Stanford WL. Systemic Mesenchymal Stromal Cell Transplantation Prevents Functional Bone Loss in a Mouse Model of AgeRelated Osteoporosis. Stem cells translational medicine. 2016;5(5):683-93.

20. Huang $S, X u L$, Sun Y, Lin S, Gu W, Liu Y, et al. Systemic Administration of Allogeneic Mesenchymal Stem Cells Does Not Halt Osteoporotic Bone Loss in Ovariectomized Rats. PLOS ONE. 2016;11(10):e0163131.

21. Li C, Laudier D, Schaffler M, editors. Remobilization restores cancellous bone mass but not microarchitecture after long term disuse in older adult dogs. Transactions 
of the 48th Annual Meeting of the Orthopaedic Research Society, New Orleans, LA; 2003.

22. Kim HA, Clement PJ, Cunningham JL. Investigation of cancellous bone architecture using structural optimisation. Journal of Biomechanics. 2008;41(3):629-35.

23. Cunningham J, Smith T, Blunn G, Goodship A, editors. Following damage, trabecular architecture is not restored by functional loading. Transactions 51st Annual Meeting of the Orthopaedic Research Society; 2005: University of Bath.

24. Sheyn D, Shapiro G, Tawackoli W, Jun DS, Koh Y, Kang KB, et al. PTH Induces Systemically Administered Mesenchymal Stem Cells to Migrate to and Regenerate Spine Injuries. Molecular therapy : the journal of the American Society of Gene Therapy. 2016;24(2):318-30.

25. Kim D, Cho SW, Her SJ, Yang JY, Kim SW, Kim SY, et al. Retrovirus-Mediated Gene Transfer of Receptor Activator of Nuclear Factor-kB-Fc Prevents Bone Loss in Ovariectomized Mice. Stem Cells. 2006;24(7):1798-805. 


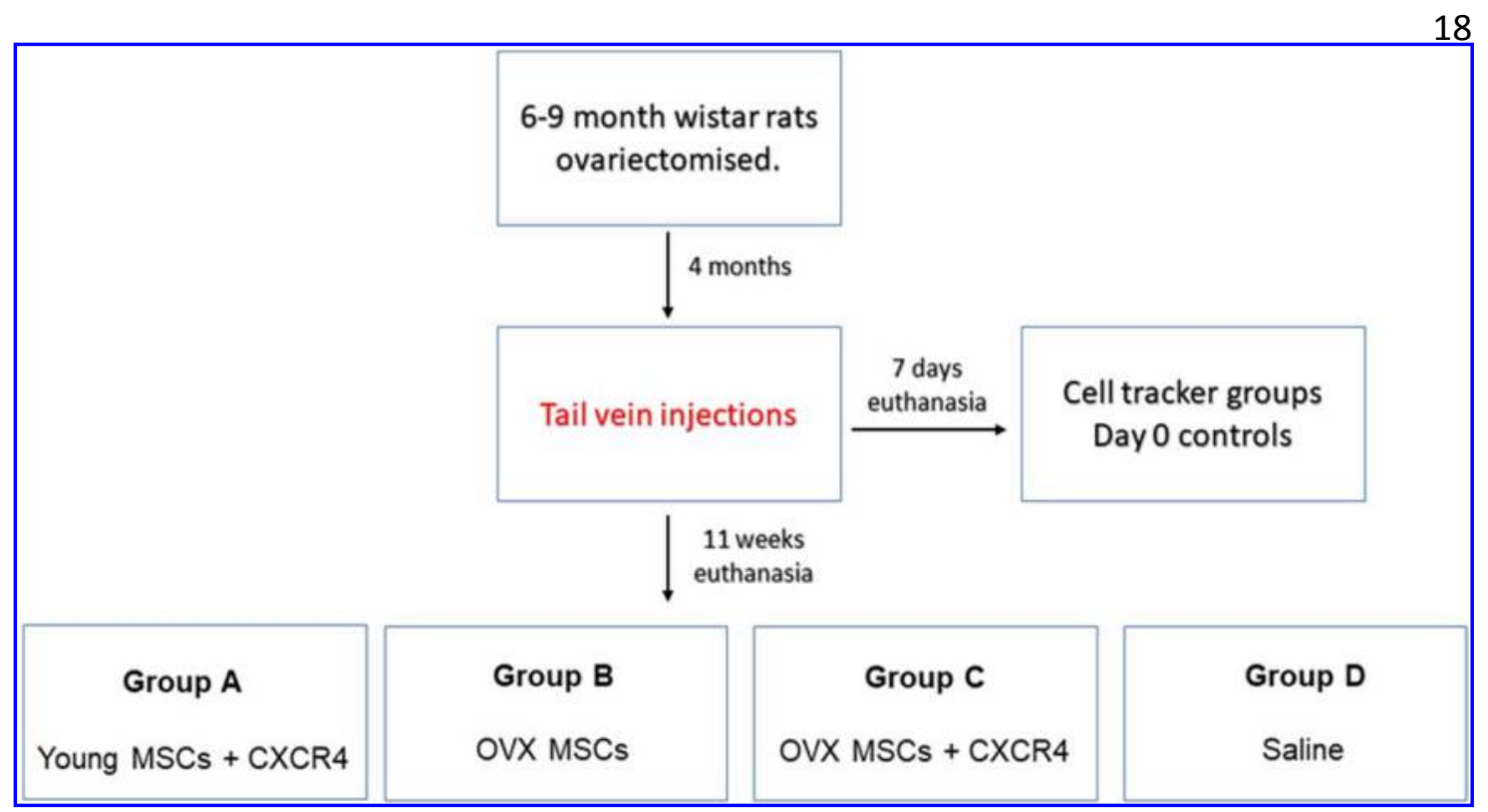

Figure 1: A schematic illustration showing the different groups of rats in the in vivo study. Cell tracker group (day 0 controls), were euthanised after 7 days, while the rest of the rats were euthanised after 11 weeks. 


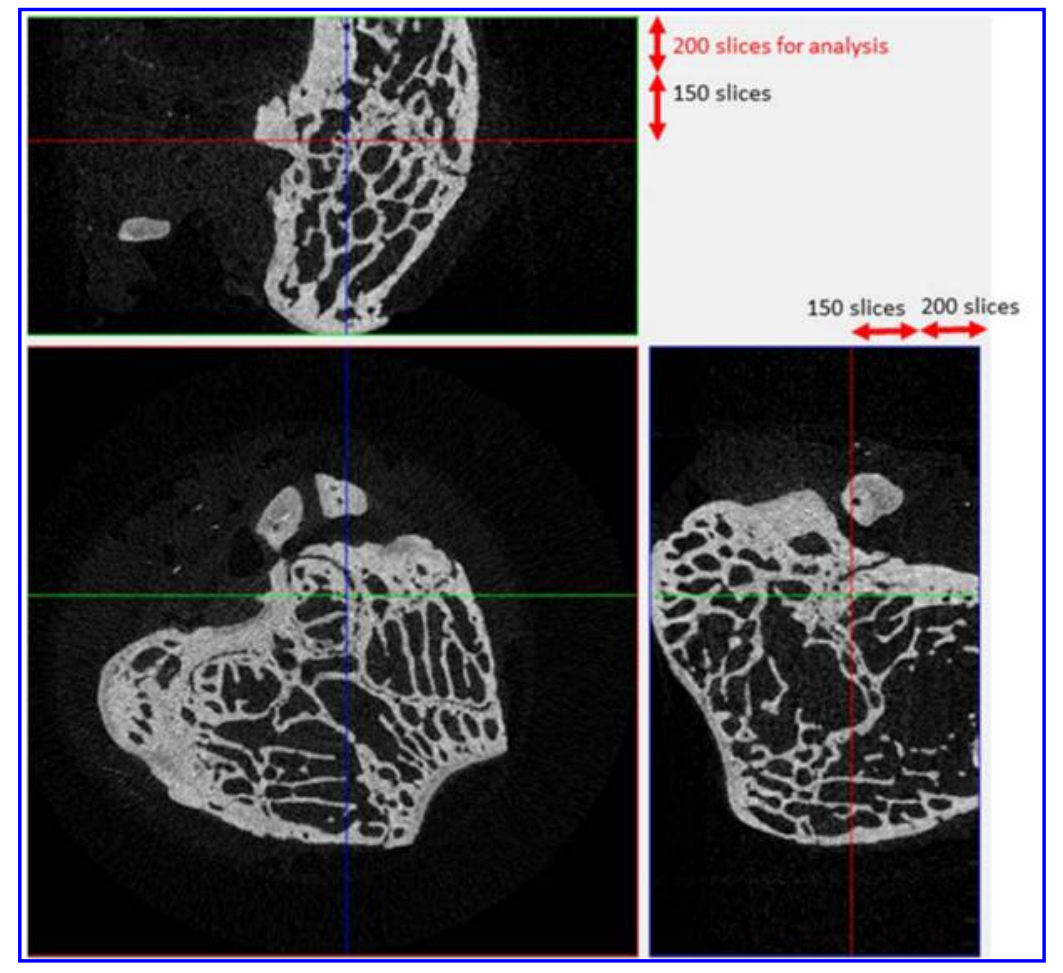

Figure 1: The sagittal, coronal and transaxial display of a reconstructed image of the growth plate, whereby a reference point was selected for analysis of bone morphometry. To avoid the growth plate, analysis was done 150 slices above the growth plate. 


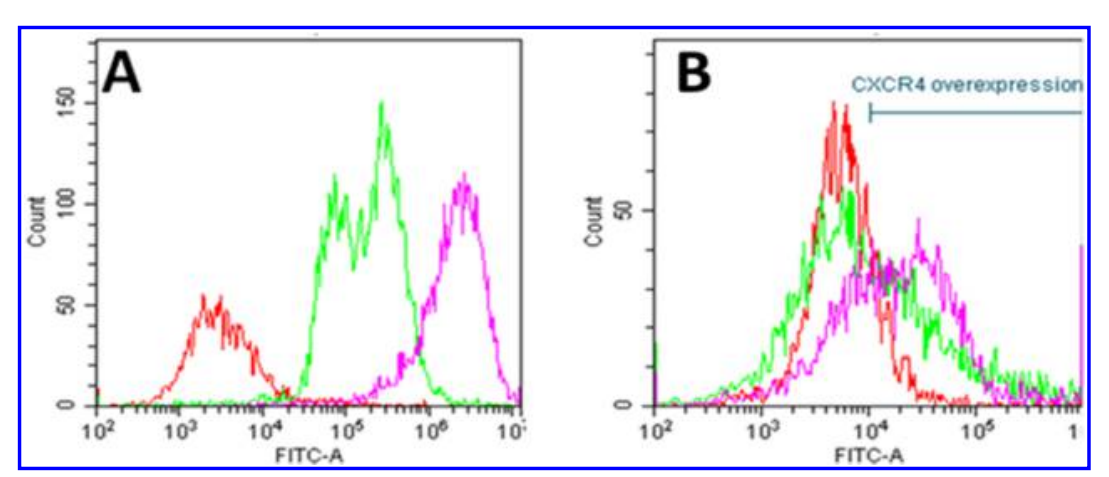

Figure 2: FACS analysis of CXCR4 infected cells and uninfected cells of young MSCs (a); adult control (b) and OVX MSCs (c). The secondary isotype is represented by red, CXCR4 expression in MSCs is represented by green and pink represents the CXCR4 overexpression in the infected MSCs 


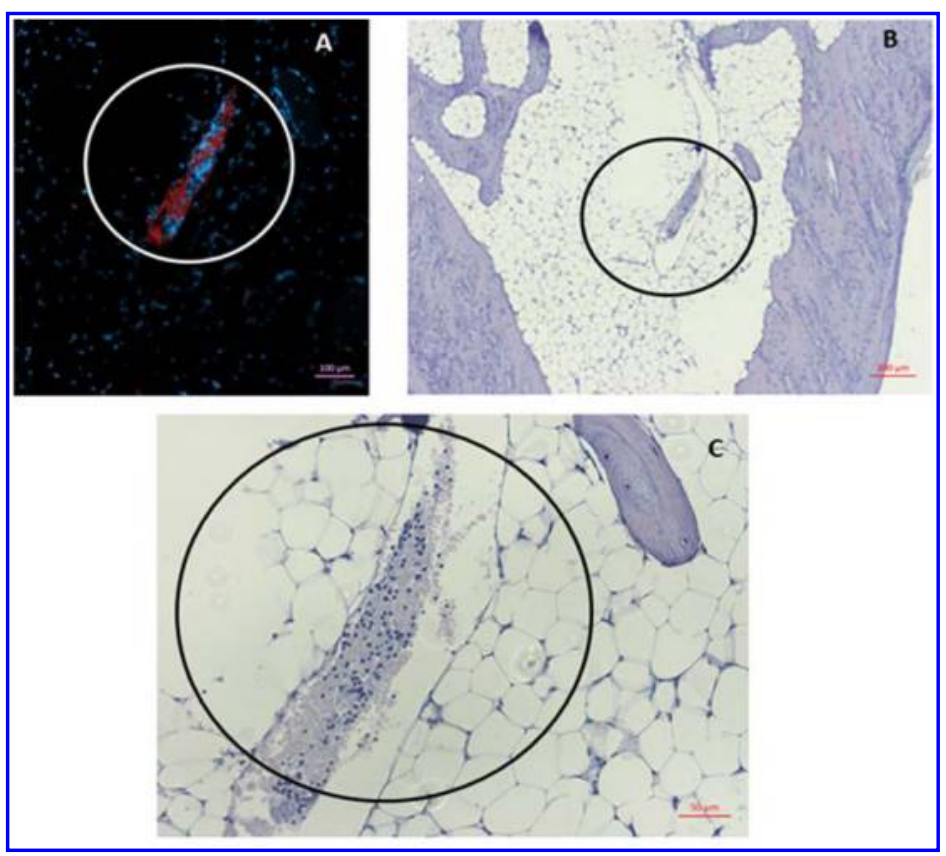

Figure 3: Dil labelled cells located in the femur (A), H \& E staining of these cells of wax histology slides of the femur (B \& C). 


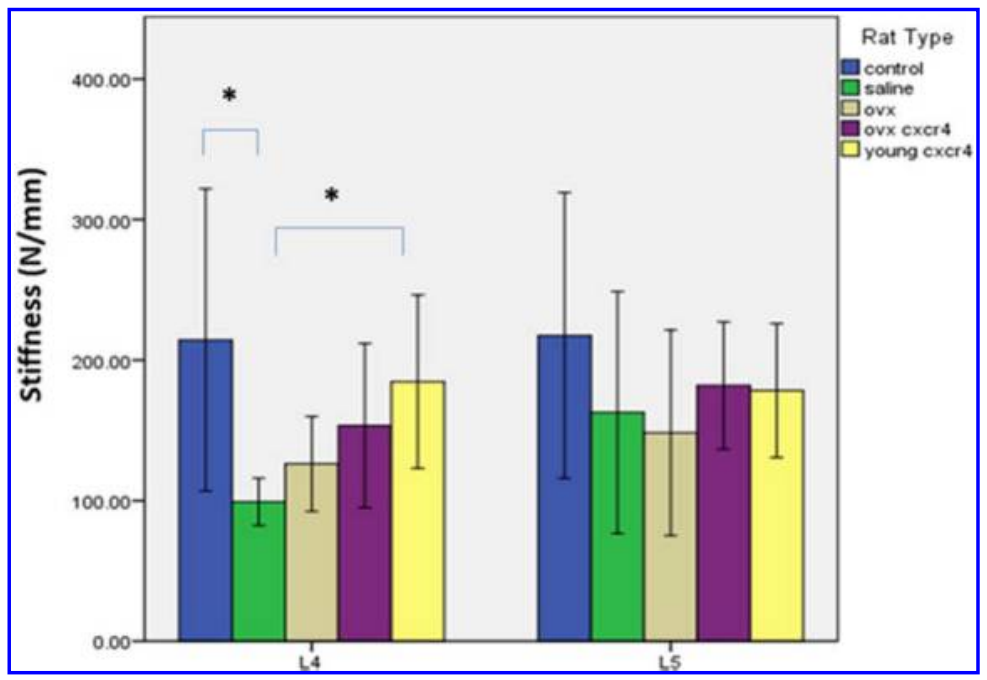

Figure 4: The stiffness of the L4 and L5 vertebrae calculated from the gradient of the compression graph $(n=6)$. Significant differences were found between Young-CXCR4 and Saline groups; as well as between Control and Saline groups. * shows significance $p<0.05$ 


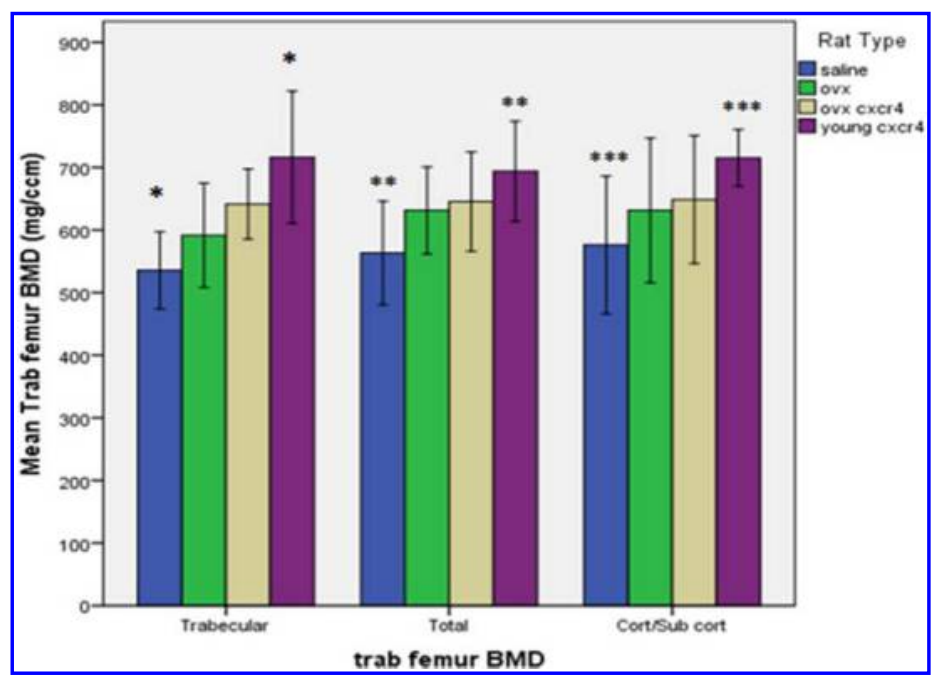

Figure 5: The mean BMD of the femur measured using PQCT. The BMD was measured for the femur and tibia and trabecular, cortical and total bone density was analysed for both bones. Difference was found between Young-CXCR4 and Saline groups in *trabecular bone, ${ }^{* *}$ cortical bone, ${ }^{* *}$ and for total bone density. 


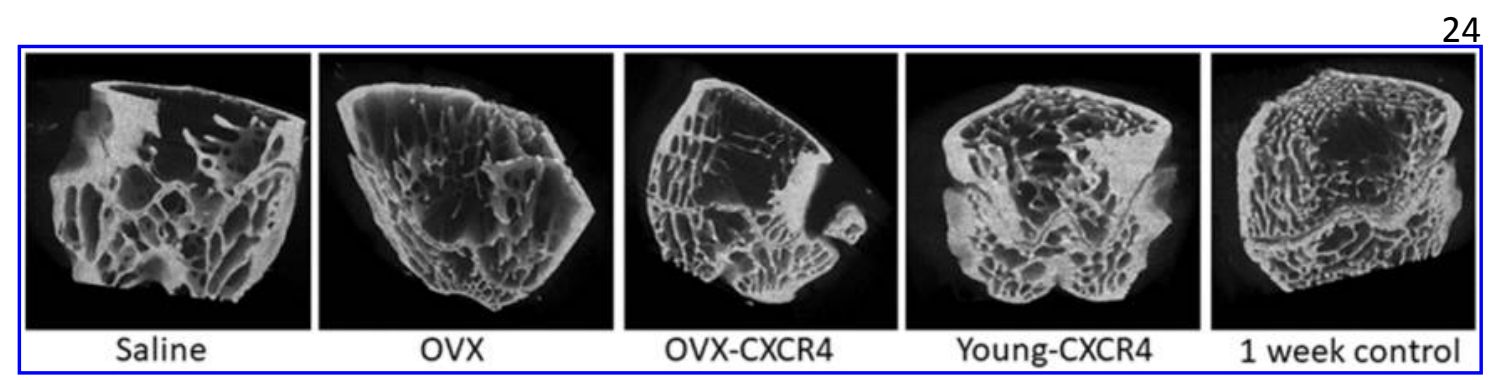

Figure 6: Three dimensional images of the femoral heads to illustrate the trabeculae structure of the metaphysis and epiphysis. 


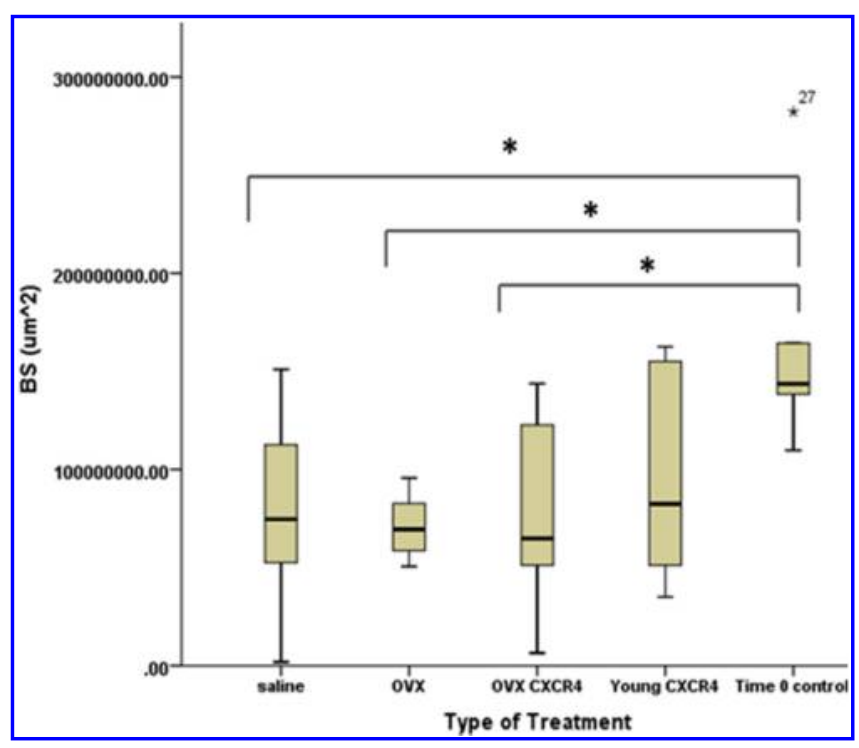

Figure 7: Bone Surface $\left(\mu \mathrm{m}^{2}\right)$ of the femur obtained from rats treated with saline, OVX MSCs, OVX-CXCR4 MSCs and young CXCR4 MSCs. The error bars represent standard error of the mean. 


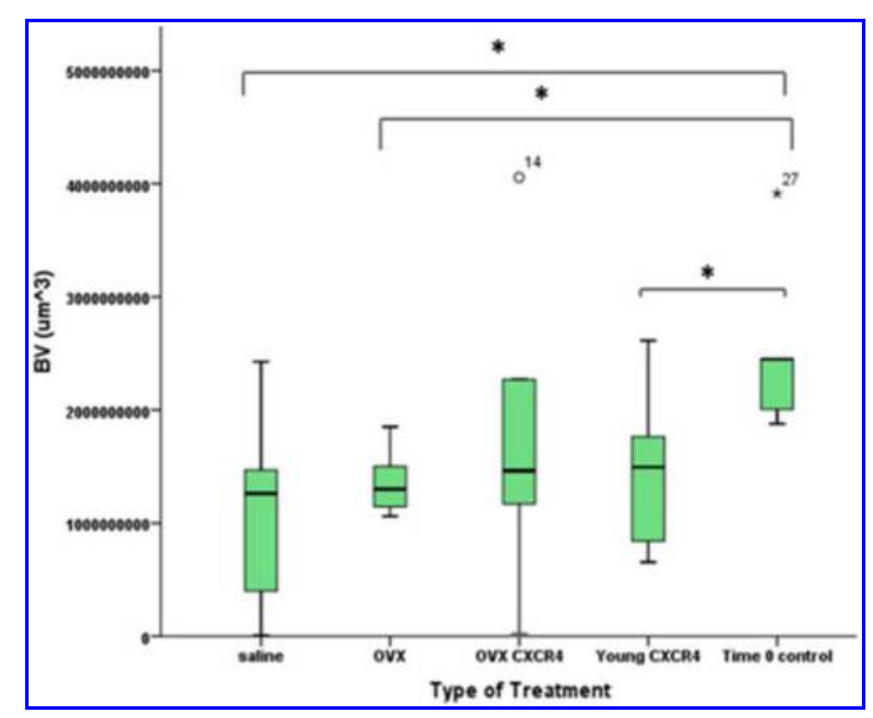

Figure 8: Bone Volume $\left(\mu \mathrm{m}^{3}\right)$ of the femur obtained from rats treated with saline, OVX MSCs, OVX-CXCR4 MSCs and young CXCR4 MSCs. The error bars represent standard error of the mean 